\title{
PRIMEIRO REGISTRO DE FÓSSEIS DE MAMÍFEROS PLEISTOCÊNICOS EM CAVERNA DE SERGIPE, BRASIL
}

\author{
MÁRIO ANDRÉ TRINDADE DANTAS \\ Centro da Terra-Grupo Espeleológico de Sergipe, Rua Antônio Teles da Costa, 42, 49045-070, Aracaju, SE, Brasil. \\ matdantas@yahoo.com.br
}

\begin{abstract}
FIRST OCCURRENCE OF LATE PLEISTOCENE-HOLOCENE MAMMALS IN CAVE IN SERGIPE STATE, BRAZIL. This paper reports the first discovery of fossils of the Late Pleistocene-Holocene mammals in caves in Sergipe State, Brazil. In recent surveys conducted in the cave Toca da Raposa, Simão Dias municipality, fossils of Glyptodon clavipes Owen (Glyptodontidae) and Galea spixii (Wagler) (Caviidae) were found. With these additions, the pleistocenic mammalian fauna in Sergipe is composed of the following taxa: Eremotherium laurillardi, Glossotherium sp., Catonyx cuvieri, Glyptodon clavipes, Smilodon populator, Toxodontinae, Stegomastodon waringi, Equinae, Palaeolama major and Galea spixii.
\end{abstract}

Key words: Toca da Raposa, upper Pleistocene-Holocene, Simão Dias, Sergipe, Brazil.

RESUMO - O presente trabalho relata a primeira descoberta de fósseis de mamíferos do Pleistoceno final-Holoceno em cavernas no Estado de Sergipe. Em recentes pesquisas realizadas na caverna Toca da Raposa, localizada no município de Simão Dias, foram encontrados fósseis de Glyptodon clavipes Owen (Glyptodontidae) e Galea spixii (Wagler) (Caviidae). Com esses dois novos registros, a fauna de mamíferos pleistocênicos de Sergipe fica composta pelos seguintes táxons: Eremotherium laurillardi, Glossotherium sp., Catonyx cuvieri, Glyptodon clavipes, Smilodon populator, Toxodontinae, Stegomastodon waringi, Equinae, Palaeolama major e Galea spixii.

Palavras-chave: Toca da Raposa, Pleistoceno final-Holoceno, Simão Dias, Sergipe, Brasil.

\section{INTRODUÇÃO}

O longo histórico das pesquisas paleontológicas em Sergipe demonstra que o Estado é rico em fósseis, principalmente do período Cretáceo e da época Pleistocênica (Souza Lima et al., 2002; Dantas, 2008). A primeira descoberta de fósseis em cavernas sergipanas ocorreu em 1997, quando um grupo de espeleólogos encontrou na Gruta da Raposa em Laranjeiras, em associação, um dente de tubarão, algumas vértebras de pequenos peixes, equinóides e gastrópodes fósseis. O dente foi posteriormente descrito por Carvalho \& Gallo (2002), que o identificaram como pertencente ao gênero Ptychodus, um tubarão que viveu durante o final do Cretáceo.

Ainda em 1997, fósseis pleistocênicos foram encontrados em outra cavidade, no Abismo de Simão Dias, no município homônimo. Nesta caverna, do tipo abismo, há um lençol freático (a altura total da boca da caverna até o lençol freático é de $42 \mathrm{~m}$ ), onde foi encontrada, em uma fenda próximo à lâmina de água, a carapaça de um Testudinidae do gênero Chelonoidis (=Geochelone; Lôbo et al., 2007). No entanto, ainda não havia registros da ocor- rência de fósseis de mamíferos nestas cavidades. Até então, toda a fauna de mamíferos registrada no Estado era procedente de afloramentos do tipo tanque (Souza-Cunha et al., 1985; Goes, 2002; Dantas, 2004; Dantas et al., 2005; Dantas \& Zucon, 2007; Dantas, 2008). Portanto, é registrado aqui a primeira ocorrência de Galea spixii e Glyptodon clavipes no município de Simão Dias, em Sergipe.

\section{MATERIAL E MÉTODOS}

O material estudado foi coletado em uma caverna calcária localizada no município de Simão Dias, Sergipe, denominada Toca da Raposa (Figura 1), estando depositado na coleção científica do Laboratório de Paleontologia da Universidade Federal de Sergipe (LPUFS). A caverna está localizada na Fazenda Manoel Roque (1044'18'S, $\left.37^{\circ} 48^{\prime} 40^{\prime \prime} \mathrm{W}\right)$.

A taxonomia dos Cingulata segue Porpino et al., (2004) e a dos Rodentia segue Wilson \& Reeder (2005). A nomenclatura dentária dos Caviidae está de acordo com Contreras (1964). 


\section{SISTEMÁTICA}

\author{
CINGULATA Illiger, 1811 \\ GLYPTODONTOIDEA Gray, 1869 \\ GLYPTODONTIDAE Gray, 1869 \\ GLYPTODONTINAE Gray, 1869 \\ Glyptodon clavipes Owen, 1839
}

(Figura 2A-B)

Material. Fragmento de molariforme LPUFS 3523; osteodermos LPUFS 3524 e 3525.

Descrição. $O$ fragmento de molariforme LPUFS 3523 (Figura 2A) apresenta as características sugeridas por Paula Couto (1979) para a Subfamília Glyptodontinae. É um dente trilobulado, com cristas de osteodentina e ramificações secundárias, estando ausente a sua face de oclusão, fraturada durante a coleta.

Nos osteodermos LPUFS 3524 (Figura 2B) e LPUFS 3525 podem ser observadas a porção exterior (ornamentada) e a região do tecido esponjoso, estando ausente a porção interna. São pentagonais, com uma figura central que apresenta no seu centro uma depressão, circundada por pequenas figuras periféricas, cujo número varia de sete a nove. Estas características são definidas por Porpino (1999) como diagnósticas de G. clavipes.

Discussão. Aos Glyptodontoidea pode ser atribuída a fórmula dentária $\mathrm{I}^{0}, \mathrm{C}^{1}, \mathrm{PM}^{4}, \mathrm{M}^{3} / \mathrm{I}_{0}, \mathrm{C}_{1}, \mathrm{PM}_{4}, \mathrm{M}_{3}$. Seus molariformes são trilobulados, sem raízes e de crescimento contínuo (Hoffstetter, 1958). Os gliptodontes eram animais herbívoros, pastadores, adaptados a ambientes abertos, como atestam sua dentição hipsodonte (Bargo et al., 2006). No
Brasil ocorrem duas espécies pleistocênicas pertencentes à subfamília Gliptodontinae: Glyptodon clavipes e Glyptodon reticulatus, ambas descritas para achados no Nordeste do Brasil (Porpino, 1999). Em Sergipe, fósseis de G. clavipes já foram encontrados no município de Gararu, em um tanque na localidade Fazenda Charco, em associação com outros oito táxons (Dantas et al., 2005).

RODENTIA Bowdich, 1821

HYSTRICOMORPHA Brandt, 1855

HYSTRICOGNATHI Brandt, 1855

CAVIIDAE Fischer de Waldheim, 1817

CAVIINAE Fischer de Waldheim, 1817

Galea spixii (Wagler, 1831)

(Figura 3A-B)

Material. LPUFS 3522, fragmento de dentário direito.

Descrição. A peça LPUFS 3522 (Figura 3) é um fragmento do dentário, com parte do incisivo $\left(\mathrm{I}_{1}\right)$, e os molares $\left(\mathrm{M}_{1}, \mathrm{M}_{2}, \mathrm{M}_{3}\right)$ presentes. Os molares apresentam as características atribuídas a Galea (Contreras, 1964; Ubilla \& Rinderknecht, 2001), são hipsodontes, biprismáticos, de seção sub-triangular, com fundo da fenda preenchido por cimento, tanto na fenda fundamental externa (muito profunda), como na fenda secundária interna (pouco profunda). A camada de esmalte não é contínua, estando presente nas faces mesial, distal e labial, sendo que na face lingual está restrita à fenda secundária interna.

Discussão. Os membros da família Caviidae apresentam fórmula dentária $\mathrm{I}^{1}, \mathrm{C}^{0}, \mathrm{PM}^{1}, \mathrm{M}^{1-3} / \mathrm{I}_{1}, \mathrm{C}_{0}, \mathrm{PM}_{1}, \mathrm{M}_{1-3}$. Os dentes são de crescimento contínuo, apresentando dois prismas de

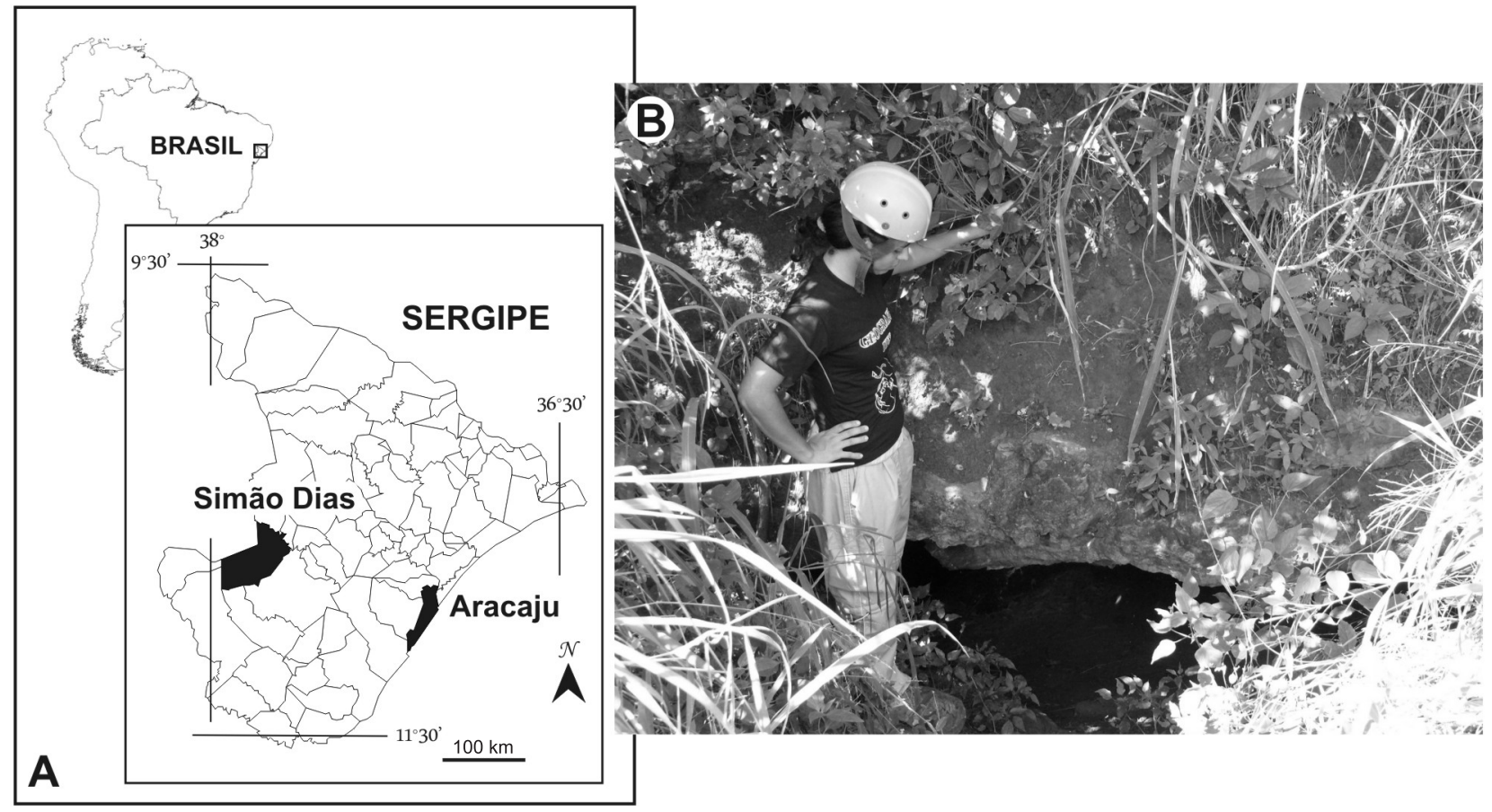

Figura 1. A, localização do município de Simão Dias, Sergipe; B, entrada da caverna Toca da Raposa.

Figure 1. A, location map of Simão Dias municipality, Sergipe State; B, entrance of Toca da Raposa cave. 

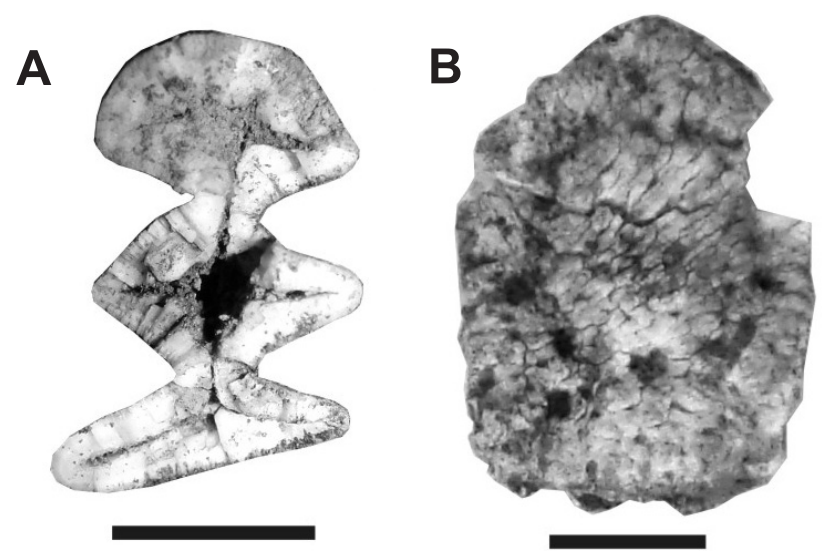

Figura 2. Glyptodon clavipes: A, molariforme LPUFS 3523; B, osteoderma LPUFS 3524 . Escalas $=10 \mathrm{~mm}$.

Figure 2. Glyptodon clavipes: A, molariform LPUFS 3523; B, osteoderm LPUFS 3524 . Scale bars $=10 \mathrm{~mm}$.

A
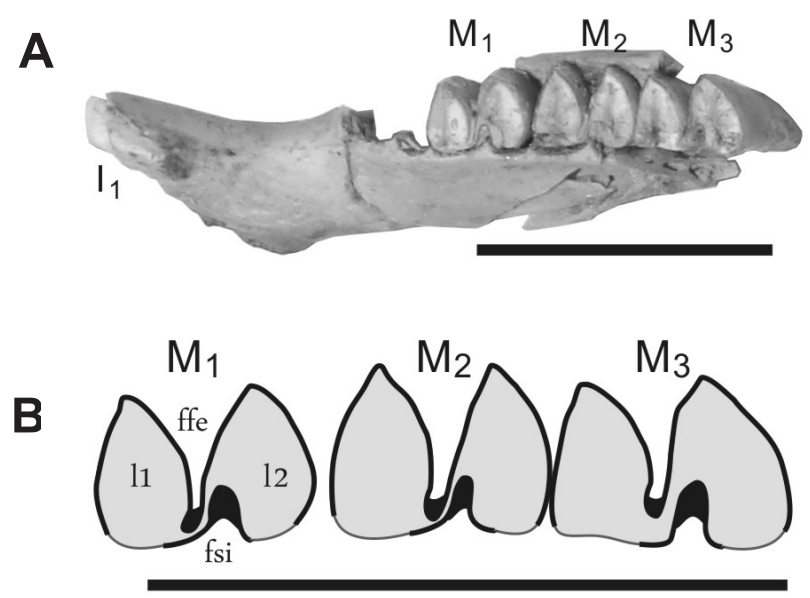

Figura 3. Galea spixii: A, corpo mandibular direito LPUFS 3522; B, desenho esquemático da vista oclusal da série $M_{1}-M_{3}$. Abreviaturas: ffe, fenda fundamental externa; fsi, fenda secundária interna; I1, primeiro prisma; $\mathbf{1 2}$, segundo prisma. Escalas $=10 \mathrm{~mm}$.

Figure 3. Galea spixii: A, right mandibular LPUFS 3522; B, oclusal view of the series $M_{1}-M_{3}$. Abbreviations: ffe, external flexus; fsi; second internal flexus; 11, first prism; 12, second prism. Scale bars $=10 \mathrm{~mm}$. esmalte, com lâminas cortantes e projeções angulares (Eisenberg \& Redford, 1999).

Esta família subdivide-se nas subfamílias Caviinae, Dolichotinae e Hydrochoerinae (Wilson \& Reeder, 2005). No Brasil ocorrem dois gêneros pertencentes à subfamília Caviinae: Cavia, com seis espécies, e Galea, com duas espécies, G. musteloides e G. spixii (Wilson \& Reeder, 2005), pois de acordo com Paula Couto (1979), G. flavidens seria sinônimo júnior de G. spixii. Galea spixii é um roedor (preá) que existe nos dias atuais, adaptado a ambientes abertos, vivendo em caatingas e cerrados brasileiros, tendo gramíneas como principal fonte de alimentação (Mares \& Lancher Jr, 1987; Eisenberg \& Redford, 1999; Reis et al., 2006). Fósseis desta espécie já foram encontradas na Argentina, Bolívia e Uruguai (Ubilla \& Rinderknecht, 2001). No Brasil foram descritas por Winge em 1888, baseado nos achados de Peter Lund em cavernas de Lagoa Santa (Minas Gerais), sendo atribuídas ao Pleistoceno, com dúvidas, por Paula Couto (1979).

Baseado nos caracteres diagnósticos do gênero, e na distribuição geográfica das espécies de Galea, atribui-se o fóssil estudado à espécie Galea spixii.

\section{CONSIDERAÇÕES FINAIS}

O fóssil de Galea spixii foi encontrado na parede de um corredor, que dá acesso a um pequeno salão onde foram encontrados, no teto, fósseis de Glyptodon clavipes. A matriz era arenosa, de coloração alaranjada, apresentando clastos de tamanhos variados (Figura 4).

As camadas fossilíferas foram, provavelmente, formadas em dois momentos distintos por eventos de enxurrada, em que o material sofreu relativamente pouco transporte, da área de origem até este local de deposição. Tal fato pode ser explicado pela presença de grandes clastos associados aos ossos cranianos de G. spixii e G. clavipes.

Como acima discutido, os achados de G. clavipes no Brasil são atribuídos ao Pleistoceno final (Porpino et al., 2004), enquanto os fósseis de G. spixii, espécie ainda vivente, foram atribuídos ao Pleistoceno, com dúvidas, por Paula Couto (1979), para restos oriundos de Minas Gerais. Como não foi possível fazer uma datação do material coletado, por prudência, estes restos são por ora atribuídos ao Pleistoceno finalHoloceno.
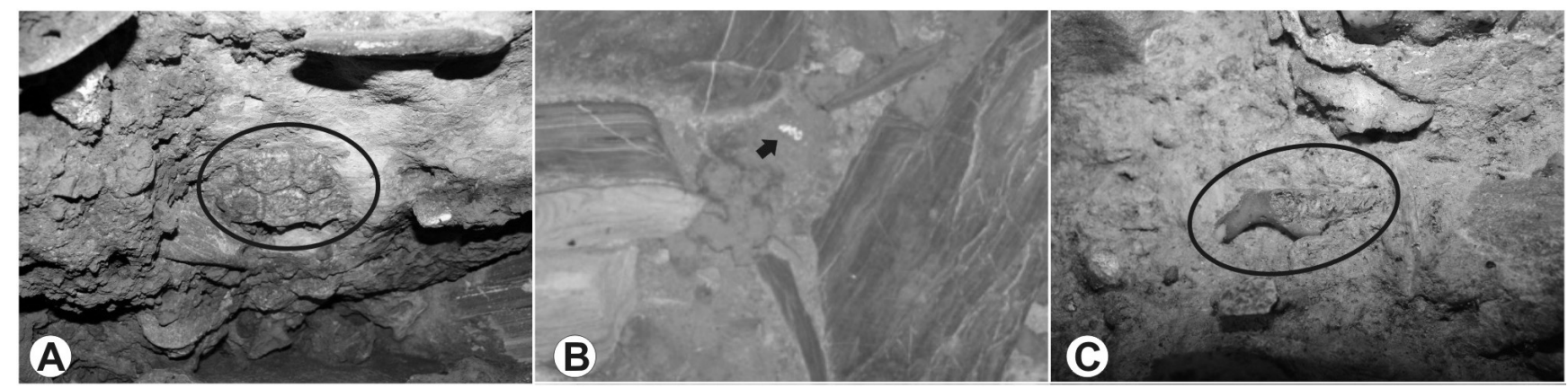

Figura 4. Camadas fossilíferas. A, osteodermos de G. clavipes; B, molariforme de G. clavipes; C, fragmento do dentário de G. spixii.

Figure 4. Fossiliferous beds. A, osteoderms of G. clavipes; B, molariform of G. clavipes; C, right mandibular of $G$. spixii. 


\section{AGRADECIMENTOS}

O autor agradece a R. Lopes (UFLA) pela descoberta dos fósseis na caverna Toca da Raposa; a A. Queiroz (UFS) pelo empréstimo de material de comparação, e pelo auxílio na identificação do espécime fóssil de Galea spixii; a P. Hadler (UFRPE) pela disponibilização de artigos referente aos Caviidae; aos dois revisores anônimos pela revisão crítica do manuscrito.

\section{BIBLIOGRAFIA}

Bargo, M.S.; De Iuliis, G. \& Vizcaíno, S.F. 2006. Hypsodonty in Pleistocene ground sloths. Acta Palaeontologica Polonica, 51(1):53-61.

Carvalho, M.S.S. \& Gallo, V. 2002. The presence of Ptychodus (Chondrichthyes, Hybodontoidea) in the Cotinguiba Formation, upper Cretaceous of the Sergipe-Alagoas basin, northeastern Brazil. In: SIMPÓSIO SOBRE O CRETÁCEO DO BRASIL, 6, 2002. Boletim de Resumos, São Pedro, UNESP, p. 307-309.

Contreras, J.R. 1964. Datos acerca de la variación intrapoblacional de la morfología de los molares de entidades de los gêneros Galea y Microcavia (Rodentia, Caviidae). Ameghiniana, 3(8):235-255.

Dantas, M.A.T. 2004. Os fósseis da megafauna pleistocênica do Instituto Histórico e Geográfico de Sergipe. Canindé, 4:383-393.

Dantas, M.A.T. 2008. Paleomastozoologia sergipana: as descobertas na localidade Sítios Novos, Canhoba, Sergipe, Brasil. Revista de Geologia, 21(2):159-168.

Dantas, M.A.T.; Zucon. M.H. \& Ribeiro, A.M. 2005. Megafauna pleistocênica de Gararu, Sergipe, Brasil. Revista de Geociências (UNESP), 24(3):277-287.

Dantas, M.A.T. \& Zucon, M.H. 2007. Occurrence of Catonyx cuvieri (Lund, 1839) (Tardigrada, Scelidotheriinae) in Late Pleistocene-Holocene of Brazil. Revista Brasileira de Paleontologia, 10(2):129-132.

Eisenberg, J.F. \& Redford, K.H. (eds.) 1999. Mammals of the Neotropics. Chicago, The University of Chicago Press, 609 p.

Goes, F.A.S.; Vieira, F.S.; Zucon, M.H.; Cartelle, C. \& Teodósio, C. 2002. Ocorrência de mamíferos pleistocênicos em Sergipe, Brasil. Arquivos do Museu Nacional, 60(3):199-206.
Hoffstetter, R. 1958. Xenarthra. In: J. Piveteau (ed.) Traité de Paléontologie, Masson, p. 535-626.

Lôbo, D.; Silva, E.J.; Carvalho, J.L.G.; Santos, L.C.M.; Santana, M.O.; Leão, T.C.C.; Zucon, M.H. \& Valerio, M.E.G. 2007. Ocorrência de Geochelone (Reptilia-Testudinidae) no Abismo de Simão Dias, Sergipe, Brasil. Revista Direto do Centro da Terra, 1(1):5-8.

Mares, M.A. \& Lancher Jr., T.E. 1987. Ecological, morphological and behavioral convergence in rock-dweling mammals. In: H.H. Genoways (ed.) Current Mammology, Plenum Publishing Corporation, p. 307-348.

Paula Couto, C. de. 1979. Tratado de Paleomastozoologia. Rio de Janeiro, Academia Brasileira de Ciências, 590 p.

Porpino, K.O. 1999. Estudo dos Cingulata (Xenarthra, Mammalia) fósseis depositados no Museu Câmara Cascudo, Natal-RN. Programa de Pós-Graduação em Zoologia, Universidade Federal do Rio de Janeiro, Dissertação de Mestrado, 138 p.

Porpino, K.O.; Santos, M.F.F. \& Bergqvist, L.P. 2004. Registros de mamíferos fósseis no Lajedo de Soledade, Apodi, Rio Grande do Norte, Brasil. Revista Brasileira de Paleontologia, 7(3):349-358.

Reis, N.R. dos; Peracchi, A.L.; Pedro, W.A. \& Lima, I.P. de. 2006. Mamíferos do Brasil. Londrina, 437 p.

Souza-Cunha, F.L.; Andrade, A.B.; Zucon, M.H. \& Santos, M.M 1985. Ocorrência de mamífero fóssil pleistocênico localizado em Monte Alegre, Sergipe, Brasil. Coletâneas de Trabalhos Paleontológicos (DNPM), 7(2):29-33.

Souza-Lima, W.; Andrade, E.J.; Bengston, P. \& Galm, P.C. 2002. A Bacia de Sergipe-Alagoas: evolução geológica, estratigráfica e conteúdo fóssil. Aracaju, Fundação Paleontológica Phoenix, 34 p.

Ubilla, M. \& Rinderknecht, A. 2001. Consideraciones sobre el género Galea Meyen, 1831 (Rodentia, Caviidae), su registro en el Pleistoceno de Uruguay y descripción de una nueva especie extinguida. Boletín de la Real Sociedad Española de Historia Natural (sección geológica), 96:111-122.

Wilson, D.E. \& Reeder, D.M. 2005. Mammal species of the world: a taxonomic and geographic reference. $3^{\mathrm{a}}$ ed. Baltimore, Maryland, John Hopkins University Press, 2142 p.

Winge, H. 1988. Jordfundne og Nulevende gnavere (Rodentia) fra Lagoa Santa, Minas Geraes, Brasilien. E. Museo Lundii, 3: 1-178.

Received in July, 2008; accepted in May, 2009. 